\title{
Análisis espacial de la vulnerabilidad socioeconómica de la ciudad de Luján (Argentina): interpretación modelística a partir de su mapa social para la identificación de áreas prioritarias de planificación
}

\section{Spatial analysis of socio-economic vulnerability in the city of Luján (Argentina): a modelling interpretation from its social map to identify priority planning areas}

\author{
Noelia Principi \\ Doctoranda en Geografía. Magister en Sistemas de Información Geográfica y Teledetección. Instituto de Investigaciones Geográficas. \\ Universidad Nacional de Luján. Ruta Nacional N 5 y Av. Constitución, (6700) Luján, Buenos Aires, Argentina, nprincipi@unlu.edu.ar, \\ ORCID https://orcid.org/0000-0002-8819-6743 \\ Gustavo Buzai \\ Doctor en Geografía. Investigador Principal del Consejo Nacional de Investigaciones Científicas y Técnicas (CONICET). Instituto de \\ Investigaciones Geográficas. Universidad Nacional de Luján. Ruta Nacional N 5 y Av. Constitución, (6700) Luján, Buenos Aires, Argentina, \\ gdb@unlu.edu.ar, ORCID https://orcid.org/0000-0003-4195-5324
}

Recibido: 11 de mayo 2020 || Aprobado: 24 de julio 2020

\section{Resumen}

El artículo presenta el análisis de la distribución espacial de la vulnerabilidad socioeconómica en la ciudad de Luján y su interpretación modelística considerando su mapa social con la finalidad de identificar áreas de planificación. Se utilizan metodologías de análisis multivariado mediante el uso de Sistemas de Información Geográfica para la construcción de áreas diferenciales y el Análisis Exploratorio de Datos Espaciales (ESDA, Exploratory Spatial Data Analysis) que permite medir la correlación como indicador de la asociación espacial entre ambos componentes. La importancia de esta línea de aplicaciones radica en generar herramientas de la Geografía Aplicada en apoyo al Ordenamiento Territorial.

Palabras clave: Vulnerabilidad socioeconómica; Mapa Social; Análisis Espacial; Geografía Aplicada; Ciudad de Luján

Abstract

The paper presents an analysis of the spatial distribution of socioeconomic vulnerability in the city of Luján and an interpretation, based on a model using a social map of the city, to identify planning areas. Multivariate analysis is combined with Geographical Information Systems (GIS) for the identification of differentiated areas and Exploratory Analysis of Spatial Data (ESDA) allows to meassure the correlation as an indicator of the spatial association between both components. This type of application emphasizes the significance of the tools of Applied Geography in support of Territorial Planning.

Key words: Socioeconomic vulnerability; Social Map; Spatial Analysis; Applied Geography; City of Luján

Cita sugerida: Principi, N. y Buzai, G. (2020). Análisis espacial de la vulnerabilidad socioeconómica de la ciudad de Luján (Argentina): interpretación modelística a partir de su mapa social para la identificación de áreas prioritarias de planificación. Estudios Socioterritoriales. Revista de Geografía, (28), 064. DOI: https://doi.org/10.37838/unicen/est.28-064 


\section{INTRODUCCIÓN}

La vulnerabilidad socioeconómica de la población urbana es un tema de gran relevancia actual que permite evidenciar las desigualdades de los sectores sociales más desfavorecidos en las ciudades al momento de afrontar la ocurrencia de fenómenos de origen natural o antrópico que generan consecuencias adversas. Por esto, los estudios sobre vulnerabilidad se consideran imprescindibles para analizar situaciones de riesgos urbanos de diversa índole. En las últimas décadas la temática fue incluida fuertemente en los discursos políticos de nuestro país y de la región, como aspecto importante a considerar al momento de gestionar el territorio y definir políticas que tengan como objetivo mejorar las condiciones de vida de la población y, al mismo tiempo, minimizar las desigualdades sociales (CELADE, 2002). Desde la geografía es posible analizar diferentes aspectos de la vulnerabilidad a nivel espacial, a partir de estudiar variables sociales y económicas de la población urbana, generando información relevante para los tomadores de decisiones.

Si bien la problemática es multidimensional, el presente trabajo pone su foco de atención en la dimensión espacial del fenómeno mediante el estudio de la distribución espacial de la vulnerabilidad socioeconómica en la ciudad de Luján y su interpretación modelística a partir de su relación con el mapa social urbano ante la finalidad de identificar áreas prioritarias de planificación.

La teoría sistémica, en su desarrollo correspondiente al abordaje de la realidad como sistema complejo (García, 2006), justifica la focalización espacial como nivel de análisis específico que se apoya en las perspectivas paradigmáticas racionalista y cuantitativa y, junto a ellas, la posibilidad de utilización de métodos científicos para su abordaje.

La aplicación de procedimientos de análisis multivariado combinado con el uso de Sistemas de Información Geográfica (SIG) permite avanzar a partir de la definición de distribuciones espaciales modelizadas en la cartografía temática, lo que constituye un acercamiento inicial de gran importancia para lograr una aproximación a la estructura espacial de la información en el área de estudio. El análisis exploratorio de datos espaciales (ESDA, Exploratory Spatial Data Analysis), a través del uso de gráficos interactivos y el modelado cartográfico mediante la aplicación de procedimientos matemáticos en la tabla de atributos de un SIG vectorial, se convierten en herramientas fundamentales que permiten avanzar en el análisis de asociación espacial en la búsqueda de relaciones causales.

A lo largo del trabajo se presenta el área de estudio, el detalle de los procedimientos teórico-metodológicos de análisis multivariado: cálculo del valor índice medio (VIM) para acceder a la distribución espacial de vulnerabilidad socioeconómica y de método Linkage Analysis para la realización del mapa social. El ESDA y el modelado cartográfico permiten avanzar en sus vínculos e identificar zonas críticas como áreas prioritarias de planificación.

ÁREA DE ESTUDIO: CIUDAD DE LUJÁN

La ciudad de Luján es una aglomeración de tamaño intermedio de la República Argentina (Figura 1). Se encuentra dividida espacialmente en 87 radios censales $^{1}$ urbanos como mosaico de polígonos que la contienen y que, de acuerdo a los datos del

1 El radio censal está definido por un espacio territorial con límites geográficos y una determinada cantidad de unidades de viviendas a relevar en el Censo Nacional de Población, Hogares y Viviendas. Según el Instituto Nacional de Estadística y Censos (INDEC, 2013), en promedio, un radio censal tiene 300 viviendas, aunque hay que considerar que para bordes de localidades el radio urbano puede descender a 200 viviendas. 
Censo Nacional de Población, Hogares y Viviendas del año 2010, cuenta con 78.346 habitantes (INDEC, 2013).

Aunque su mancha urbana se separa en distancias de 1 a 2 kilómetros del extremo del corredor Oeste del Gran Buenos Aires (Humacata, 2015), la infraestructura gris (espacio adaptado + sistema de flujos) indica que forma parte de la Megaciudad Buenos Aires (Buzai y Montes Galbán, 2020).

El río Luján realiza un recorrido de 115 kilómetros por la planicie ondulada de la pampa hasta su desembocadura en el delta del Paraná (Buzai, Lanzelotti, Paso Viola y Principi, 2018). En su sector medio atraviesa la totalidad de la ciudad de Luján y sus desbordes generan inundaciones urbanas en toda su extensión, abarcando zonas céntricas y periféricas. Por este motivo, resulta significativo estudiar la vulnerabilidad socioeconómica de la población, ya que permite avanzar en el estudio espacial del riesgo en el marco de la Geografía Aplicada con el objetivo de brindar apoyo a la práctica del Ordenamiento Territorial (Phlipponneau, 2001).

Figura 1. Área de estudio. Ciudad de Luján (Buenos Aires, Argentina)

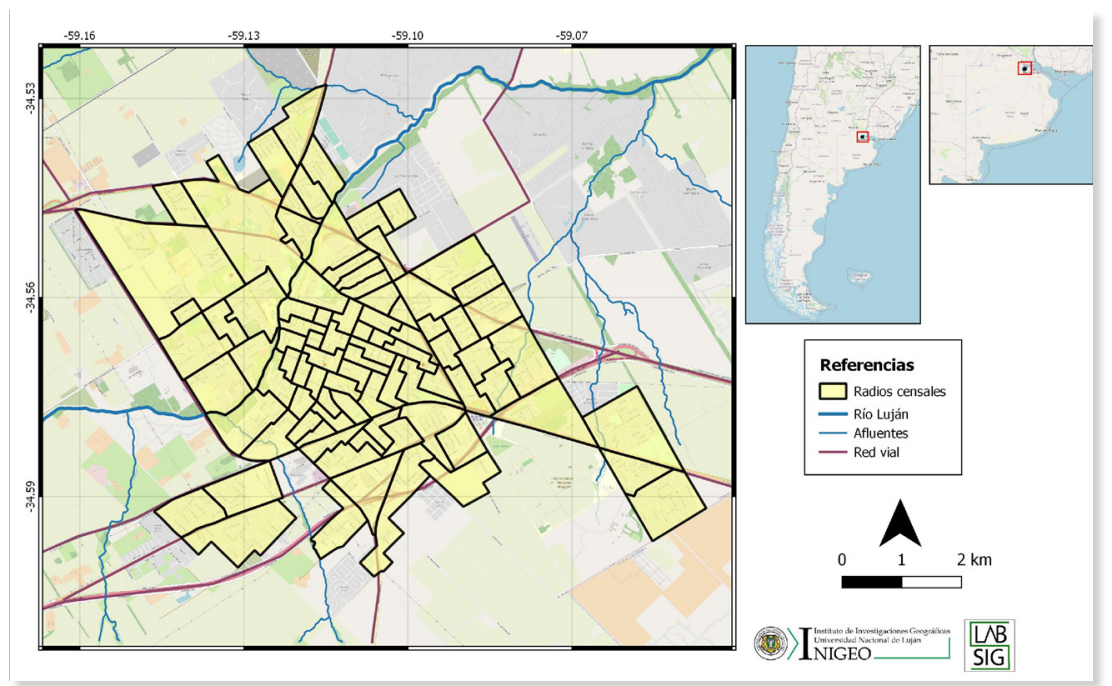

Fuente: elaboración personal con base en datos del INDEC (2013) y mapa base de Open Street Map

\section{ASPECTOS CONCEPTUALES}

El análisis realizado en la ciudad de Luján, focaliza su atención en la distribución espacial de la vulnerabilidad socioeconómica de la población y su interpretación a partir del modelo urbano representando por el mapa social de la ciudad. El abordaje se realiza desde un punto de vista sistémico (Bertalanffy, 1976) y avanza en un nivel de focalización espacial como objeto de estudio formal de la geografía en el marco de los estratos en los que se organiza la realidad como sistema complejo (García, 2006). Ambas perspectivas se complementan a partir de sus objetivos basados en la generalidad y particularidad respectivamente (Principi, 2020) y apoyadas en la teoría, las variables y las metodologías aplicadas se centran en un punto de vista espacial.

Es de destacarse que conceptualmente complejo no es equivalente a complicado, por lo tanto resulta posible la realización de estudios claramente delimitados en un determinado nivel en la estratificación de la realidad como sistema, y, en este sentido, la Geografía Cuantitativa y los SIG aportan específicamente en el nivel espacial. 
Esta es la concepción que enmarca el concepto de vulnerabilidad al hacerlo operativo en la perspectiva espacial a través de la combinación de variables que permiten evidenciar las condiciones de desigualdad que tiene la población para enfrentar la ocurrencia o probabilidad de ocurrencia de algún peligro (Principi, 2018). Actualmente, existe una gran diversidad en torno al concepto de vulnerabilidad (Egea Jiménez, Sánchez González y Soledad Suescún, 2012; Cardona, 2003). Se la puede considerar en términos de la capacidad de la sociedad para adaptarse a los cambios del ambiente, desde un punto de vista sistémico (Wilches-Chaux, 1993; Blaikie, Cannon, Davies y Wisner, 2002), también como la predisposición de un individuo o grupo social a sufrir daños (Herzer, 1990, Cardona, 1993, Lavell, 1996) o como un conjunto de condiciones de diversa índole que preceden a una amenaza de origen natural o antrópica y determinan los impactos a sufrir (Blaikie et al., 2002; CELADE, 2002). En todos los casos se considera que las características principalmente sociales y económicas de la sociedad pueden magnificar o minimizar el impacto de fenómenos amenazantes. Aun cuando existe gran cantidad de bibliografía con aspectos teóricos y aplicaciones sobre la temática, no existe un procedimiento universal para el estudio de la vulnerabilidad, debido a la naturaleza polisémica del concepto (CELADE, 2002).

Es importante mencionar el aporte conceptual realizado en el marco de la llamada Escuela de Ecología Humana de la Universidad de Chicago, con orígenes en el ámbito de la sociología, aunque con expansión hacia diferentes áreas del conocimiento, entre ellas la geografía. Desde el Departamento de Geografía de dicha universidad, a través de Barrows (1923) surgió una línea de análisis que consideró a la Geografía como ciencia central de la Ecología Humana, haciendo énfasis en investigaciones que analizaban las relaciones entre los seres humamos y su entorno natural desde un punto de vista ecológico aprovechando un campo multidisciplinario que se ocupa de la humanidad (Kates, 1971). Esta idea fue continuada en la década de 1960 por su discípulo, el geógrafo Gilbert White, considerado el precursor del análisis de riesgos y desastres en Geografía, y sus colegas Robert Kates y lan Burton, quienes incursionaron en estudios de riesgos incorporando en el análisis la respuesta y adaptación de las personas en relación a su entorno y la incertidumbre vinculada a la difícil previsión de los eventos naturales (Martínez Rubiano, 2009). White (1974) definió al riesgo como resultado de la vinculación entre el peligro y la vulnerabilidad (riesgo = peligro $x$ vulnerabilidad). De este modo, se pone énfasis en la vulnerabilidad como el factor de mayor peso en la concepción de riesgo. Esta definición contempla que ante un mismo escenario de peligro, el riesgo será mayor para aquellas personas y/o lugares que presenten una vulnerabilidad más alta.

Desde un punto de vista amplio Wilches-Chaux (1993) definió la 'vulnerabilidad global', compuesta por gran cantidad de dimensiones que la constituyen y que se pueden analizar de forma sistémica: 1) 'dimensión natural', vinculada a los límites ambientales; 2) 'dimensión física', con respecto a la localización de la población en zonas de riesgo físico; 3) 'dimensión económica', en relación a los sectores económicamente más desfavorecidos; 4) 'dimensión social', vinculada a la organización y cohesión interna de la sociedad; 5) 'dimensión política', vinculado a la toma de decisiones y la organización gubernamental; 6) 'dimensión técnica', respecto a la utilización o existencia de técnicas y tecnología adecuadas; 7) 'dimensión ideológica', vinculada a la concepción que tienen las personas del mundo y del ambiente con el que interactúan; 8) 'dimensión cultural', relacionada con la forma en que las personas se ven a sí mismas, en la sociedad y como 
un conjunto nacional, y también el papel que juegan los medios de comunicación; 9) 'dimensión educativa', vinculada al grado de preparación de la sociedad; 10) 'dimensión ecológica', con énfasis en las consecuencias de la alteración de los ecosistemas; y 11) 'dimensión institucional', vinculada a la obsolescencia y rigidez de las instituciones.

En este trabajo se consideran aspectos de la dimensión física (características sociohabitacionales), social, educacional y económica medidos a través de variables censales de tipo estructural que permiten evidenciar situaciones de vulnerabilidad socioeconómica a nivel espacial y de esta forma, es posible asociarlo espacialmente con la estructura socioespacial interna de la ciudad de Luján, midiendo la correlación con el mapa social urbano.

El estudio de la estructura socioespacial interna de las ciudades fue un tema que llamó la atención muy tempranamente a los científicos sociales y según Carter (1995) ocupa una importante tradición en los estudios de Geografía Urbana. La línea temática realiza aportes en tres instancias a lo largo del siglo XX: en su comienzo inicia con el mapa de la pobreza de Charles Booth, avanza con la propuesta de los modelos clásicos para las ciudades anglosajonas en la primera mitad del siglo y transcurre su segunda mitad con los ajustes realizados para las ciudades de América Latina (Buzai, 2014).

El modelo de los anillos concéntricos (Burgess, 1925) fue el primer modelo que apeló a la geometría como lenguaje de la forma espacial, explicaba las distribuciones internas a través de la utilización de conceptos tomados de la ecología, incorporada al estudio de la realidad urbana a través de la Ecología Urbana, un campo de investigación desarrollado por la ya mencionada Escuela de Chicago (Gottdiener, Budd y Lehlovuori, 2016). El primer esquema socio-espacial urbano se transforma en la base que permite modelizar nuevas realidades que surgían a través de evoluciones espaciales específicas.

La expansión urbana inicial fue evidente a través de un crecimiento en anillos (Burguess, 1925), a esta base se le superpusieron los sectores urbanos vinculados a las vías de comunicación que se desarrollaban entre el centro y la periferia (Hoyt, 1939) y los diversos núcleos que permitían configurar las extensas superficies urbanas mediante una jerarquía de centros internos (Harris y Ullman, 1945). Si bien podían verificarse las estructuras espaciales básicas de toda ciudad, la distribución del nivel socio-económico de la población se verificaba de manera inversa en las ciudades de América Latina, en las cuales el centro principal (CBD, Central Business District) aún cumplía un importante papel en la estructuración socio-espacial. Se proponen diferentes modelos para las ciudades de América Latina, algunos con mayor afinidad a las ciudades de tamaño intermedio como el de Griffin y Ford (1980) y la gran mayoría para las grandes ciudades que en su versión más actualizada llega a la propuesta de la 'ciudad de islas' de Janoschka (2002). Una síntesis evolutiva de la dinámica de las estructuras urbanas latinoamericanas puede encontrarse en Borsdorf (2003) y una presentación de los modelos para su análisis en Buzai (2016) actualizando las tendencias observadas de la ciudad genérica en las megaciudades en Buzai (2020).

\section{MÉTOdOS}

\section{SOFTWARE Y DATOS}

Los datos a nivel de radios censales urbanos fueron obtenidos a partir de la base de datos REDATAM realizada por el Instituto Nacional de Estadística y Censos de la República Argentina (INDEC, 2013). Los procedimientos metodológicos fueron realizados mediante una combinación de software en el campo de la geoinformática correspon- 
diente a planilla de cálculo (Excel), programa de análisis estadístico (InfoStat) y Sistema de Ayuda a la Decisión Espacial (GeoDa), todos vinculados al núcleo formado por los Sistemas de Información Geográfica (QGIS).

Los datos censales fueron sistematizados en una Matriz de Datos Originales (MDO), organizada en filas (unidades espaciales) y columnas (variables), como tablas de atributos del SIG. A partir de aquí son aplicados diversos procedimientos matemáticos que generan nuevas matrices en la búsqueda de lograr una comparabilidad perfecta entre variables, base para la aplicación de métodos de análisis multivariado con los que, según Buzai y Baxendale (2013), la geografía aporta a la etapa del diagnóstico en la práctica del Ordenamiento Territorial.

\section{DISTRIBUCIÓN ESPACIAL DE LA VULNERABIIIDAD SOCIOECONÓMICA}

La vulnerabilidad socioeconómica de la población en la ciudad de Luján fue obtenida a partir de la aplicación de la metodología de cálculo del Valor Índice Medio (VIM) (García de León, 1989; 1997) en seis variables obtenidas de diferentes dimensiones (social, habitacional, educativa y económica).

Las variables consideradas son las siguientes: Hogares con Necesidades Básicas Insatisfechas (Hog_NBI), Calidad de conexión a servicios básicos: Insuficiente (SB_Insuf), Calidad constructiva de la vivienda: Insuficiente (CCV_Insuf), Máximo nivel educativo alcanzado: primario completo (Prim_com), No lee y no escribe (No_lee) y Condición de actividad: desocupado (Desoc).

La metodología de cálculo del VIM permite agrupar las diferentes unidades espaciales según características de homogeneidad y repetitividad en las variables seleccionadas. Permite la combinación de variables para clasificar unidades espaciales a modo de regionalización, formando grupos a partir del comportamiento de las variables o indicadores que se contemplan, que en este caso responde a características socioeconómicas de la población.

Los pasos técnicos realizados para la obtención del VIM fueron los siguientes:

(1) Realización de la transformación de la MDO o MDI en una matriz de datos estandarizados (MDE) calculando el puntaje estándar en base a $z=(x-m) / d$, donde $z$ es el puntaje estandarizado, $\mathrm{m}$ y d son la media y el desvío estándar de la serie de datos. El puntaje obtenido representa unidades de desvío siendo $\mathrm{m}=0$.

(2) Clasificación de los puntajes de la MDE considerando los siguientes intervalos de clase: Clase $1(<-1)$, Clase $2(-1$ a $-0,50)$, Clase 3 (-0,50 a 0), Clase $4(0$ a 0,50), Clase $5(0,50$ a 1$)$ y Clase $6(>1)$

(3) Cálculo del VIM para cada unidad espacial como promedio de los puntajes de clasificación en base a VIM = (Sum_c / n), donde Sum_c es la sumatoria de las clasificaciones y $\mathrm{n}$ la cantidad de variables.

(4) Ordenamiento de las unidades espaciales de forma descendente en base a los resultados generados por el VIM.

(5) Clasificación de las unidades espaciales en q intervalos. Para el caso de aplicación que presenta la aplicación (seis variables) son cinco categorías de la siguiente forma: Rango 1 (5 a 6 Muy superior a la media), Rango 2 (4 a 4,99 Superior a la media), Rango 3 (3 a 3,99 en intervalo medio), Rango 4 (2 a 2,99 Inferior a la media) y Rango 5 (1 a 1,99 Muy inferior a la media). 
(6) Realización de cartografía temática de los resultados con graduación de colores que indican orden de intensidad con cinco clases. Regionalización sin contigüidad espacial.

El método VIM, utilizado previamente por García Castro y Villerías Salinas (2018) para analizar la vulnerabilidad socioeconómica, constituye una aplicación multivariada con gran claridad y facilidad de interpretación de los resultados. El método presenta su mayor aptitud en variables de tipo cuantitativas del mismo sentido general, en esta aplicación se consideran variables de costo, las que en sus máximos puntajes presentan situaciones de máxima desfavorabilidad, por lo tanto la cartografía temática indica la mayor vulnerabilidad con las tonalidades más oscuras.

\section{MAPA SOCIAL URBANO}

El mapa social de la ciudad de Luján fue obtenido a partir de la aplicación de la metodología de cálculo del Linkage Analysis propuesta por McQuitty (1957) y presentada en el análisis geográfico por Racine y Reymond (1973) en 17 variables que contemplan las dimensiones de educación, vivienda y pobreza. Las variables son Hogares con Necesidades Básicas Insatisfechas (Hog_NBI), Población con máximo nivel educativo alcanzado primario completo (Prim_com), Población con máximo nivel educativo alcanzado secundario completo (Sec_com), Población con máximo nivel educativo alcanzado tercuniv completo (Ter_com), Población en viviendas con provisión de agua por red (Agua_ red), Población en viviendas con provisión de agua por red (Agua_red), Población en viviendas con provisión de aguar por motobombeador (Agua_mot), Población en viviendas con provisión de agua manual (Agua_man), Población en régimen de tenencia de la vivienda propietario (RTV_prop), Población en régimen de tenencia de la vivienda inquilino (RTV_inq), Población en régimen de tenencia de la vivienda ocupante de hecho (RTV_oc), Población que habita en casas (Hab_cas), Población que habita en ranchos o casillas (Hab_ranc), Población que habita en departamentos (Hab_dep), Población en viviendas con descarga a red (Desc_red), Población en viviendas con descarga de cámara séptica (Desc_cam), Población en viviendas con descarga a pozo (Desc_poz) y Población en viviendas sin baño/letrina (Sin_BL).

La metodología del Linkage Analysis genera macrovariables a partir de agrupar variables según su nivel de correlación y permite mapear un puntaje de especificidad de cada grupo obtenido. Los agrupamientos generados incluirán a todas las variables en uno u otro grupo de acuerdo a sus semejanzas, por tal motivo algún agrupamiento privilegiará los vínculos entre variables de costo (ya explicada en el punto anterior) y de beneficio, las cuales presentan mejores situaciones cuanto más alto sea el puntaje.

Los pasos técnicos realizados para la obtención de macrovariables son los siguientes:

1) Realización de la matriz de correlaciones (MC) a partir de los puntajes z de la MDE. Para ello se calcula el coeficiente de correlación, $r=\left(z_{(x)}{ }^{*} z_{(y)} / n-1\right)$, donde $r$ es el coeficiente de correlación de Pearson para $z_{(x)}, z_{(y)}$ como datos estandarizados de dos variables y $n$ corresponde al total de mediciones.

2) Selección de los valores máximos de correlación positiva en cada columna de la matriz, desestimando el valor 1 de la diagonal principal.

3) Encontrar el par de variables asociadas por ese valor de correlación máxima y realizar un listado en el que quede expresado la unión de variables y el valor correspondiente. 
4) Determinar los pares recíprocos, es decir, entre qué pares de variables se producen las máximas correlaciones de forma bidireccional. De aquí surge un listado de pares recíprocos que corresponden a los núcleos de las macrovariables.

5) Determinar las variables residuales e ir vinculándolas, de acuerdo a sus coeficientes de correlación, a los diferentes pares recíprocos para ir formando la composición de cada macrovariable.

6) Obtener los puntajes de especificidad para cada grupo promediando los valores z del núcleo (par recíproco).

7) Realizar la cartografía de cada macrovariable a partir del puntaje de especificidad.

El resultado obtenido a través del Linkage Analysis corresponde a simplificar la MC obteniendo grupos de variables (Macrovariables) que metodológicamente comienzan a formarse a partir de los núcleos generados por los pares recíprocos. Posteriormente, el cálculo de la especificidad permite determinar los datos centrales hacia los cuales se unen la totalidad de variables y su mapeo permite llegar a la distribución espacial de características combinadas.

En el presente trabajo, se considera el agrupamiento que concentra las variables de beneficio, las que en sus máximos puntajes presentan situaciones de máxima favorabilidad, por lo tanto la cartografía temática indica las mejores zonas con las tonalidades más oscuras y decrece el beneficio a media que los tonos pierden intensidad.

\section{GRÁFICO DE DISPERSIÓN Y MODELADO CARTOGRÁFICO}

La relación entre ambos resultados fue medida a través de un análisis bivariado realizado en el campo del ESDA y hecho operativo a través del gráfico de dispersión (scatter diagram) en el que cada variable queda representada por un eje ortogonal $\left(90^{\circ}\right)$ y cada unidad espacial aparece como un punto de localización $x-y$, en base a sus valores de coordenadas en cada eje (Buzai y Baxendale, 2012). Los valores de las variables en el diagrama se representan en puntajes $z$ y los ejes se ubican en valor 0 con centralidad en el promedio en ambas variables. De este modo se definen cuatro espacios como cuadrantes: el inferior izquierdo (--) que representa las unidades espaciales con valores bajos en ambas variables; el superior izquierdo con bajos valores en $x$ y altos en $y(-+)$; el superior derecho con valores altos en ambas variables (++); y el inferior derecho con valores altos en $x$ y bajos en y (+-). A partir de ellos puede verse la dispersión de puntos a la recta de regresión como representación gráfica del coeficiente de correlación.

Considerando una relación de causalidad entre la vulnerabilidad socioeconómica como variable dependiente del mapa social se podrá comprobar, para el caso de la ciudad Luján, con el coeficiente $r^{2}$ el nivel de explicación que una ejerce sobre la otra y de esta manera comprobar, en el espacio local, la hipótesis general presentada.

Este vínculo basado en una perspectiva racionalista tiene su correlato ante la búsqueda de la asociación espacial que existe entre ambas distribuciones espaciales. Para ello se procede a una tabulación cruzada entre las categorías de ambos mapas que con relación inversa llega a la definición de cinco áreas prioritarias de planificación presentada en la cartografía de resultado. 


\section{RESULTADOS}

\section{DISTRIBUCIÓN ESPACIAL DE LA VULNERABILIDAD SOCIOECONÓMICA EN LA CIUDAD DE LUJÁN}

El resultado obtenido a partir de los procedimientos metodológicos correspondientes al cálculo del VIM se presenta en la Figura 2, a partir del cual queda representada la distribución espacial de la vulnerabilidad socioeconómica en la ciudad de Luján.

Figura 2. Mapa de vulnerabilidad socioeconómica de la ciudad de Luján (Buenos Aires, Argentina)

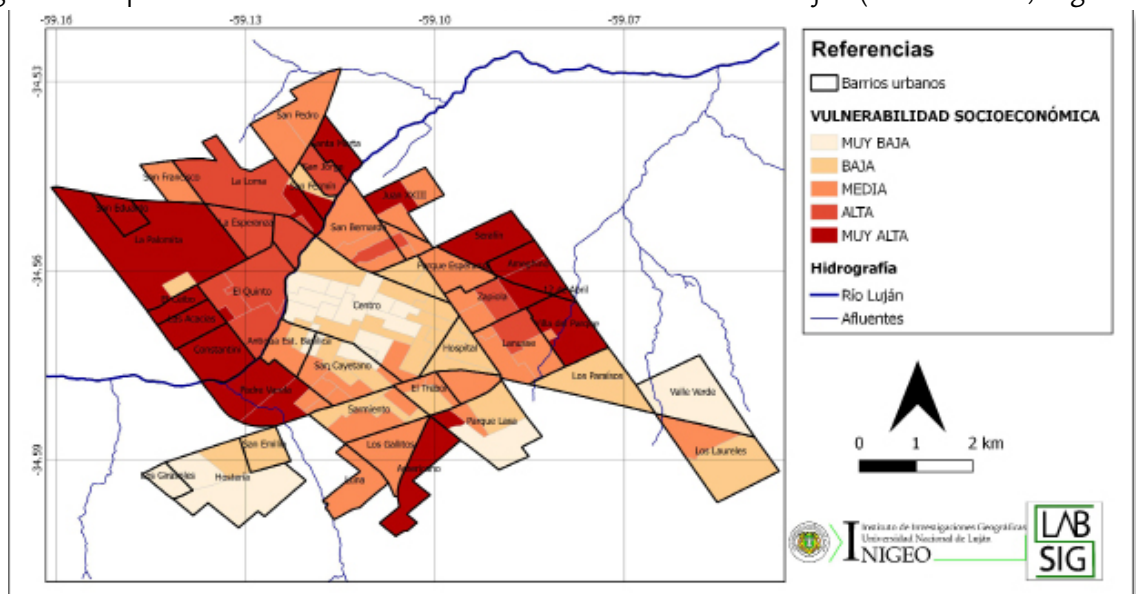

Fuente: elaboración personal con base en del Instituto Nacional de Estadística y Censos (INDEC, 2013)

Los puntajes del VIM fueron mapeados en colores graduados a fin de presentar los valores de intensidad obtenidos en cada radio censal urbano. A partir de las tonalidades puede verse un aumento escalonado de la vulnerabilidad desde el centro (color más claro) hacia la periferia (color más oscuro) en el cual se presenta, a grandes rasgos, un modelo de anillos concéntricos con dos ejes de expansión periférica de buenas condiciones (sudeste y sudoeste) en sectores de excepción en barrios que se incorporaron tardíamente al ejido urbano de la ciudad y que evolucionaron como áreas residenciales orientadas a población con nivel socioeconómico medio y medio-alto.

Los barrios que presentan condiciones de vulnerabilidad socioeconómica más desfavorables son San Eduardo, La Palomita, La Loma, La Esperanza, El Quinto, San Juan de Dios, San Fermín, San Jorge, Santa Marta, Juan XXII, Serafín, 12 de Abril, Ameghino, Zapiola, Villa del Parque, El Ceibo, Las Acacias, Constantini, Padre Varela y Americano, que corresponde en total a 28.426 habitantes (36,28\% de la población total). En contraposición, los barrios que presentan las mejores condiciones, considerando niveles muy bajos y bajos de vulnerabilidad, son el barrio Centro y barrios cercanos a este como El Mirador y Hospital a los que se suman barrios residenciales periféricos como San Emilio, Los Paraísos, Valle Verde, Hostería y Los Girasoles. Estos barrios tienen una población de 26.726 habitantes $(34,12 \%$ de la población total). Finalmente, las situaciones de vulnerabilidad media se presentan en áreas que tienen 23.194 habitantes (29,60\% de la población total).

\section{EL MAPA SOCIAL DE LA CIUDAD DE LUJÁN}

El resultado obtenido a partir de los procedimientos metodológicos correspondientes a la aplicación del Linkage Analysis se presenta en la Figura 3, a partir del cual queda representado el mapa social de la ciudad de Luján. 
Figura 3. Mapa social de la ciudad de Luján (Buenos Aires, Argentina)

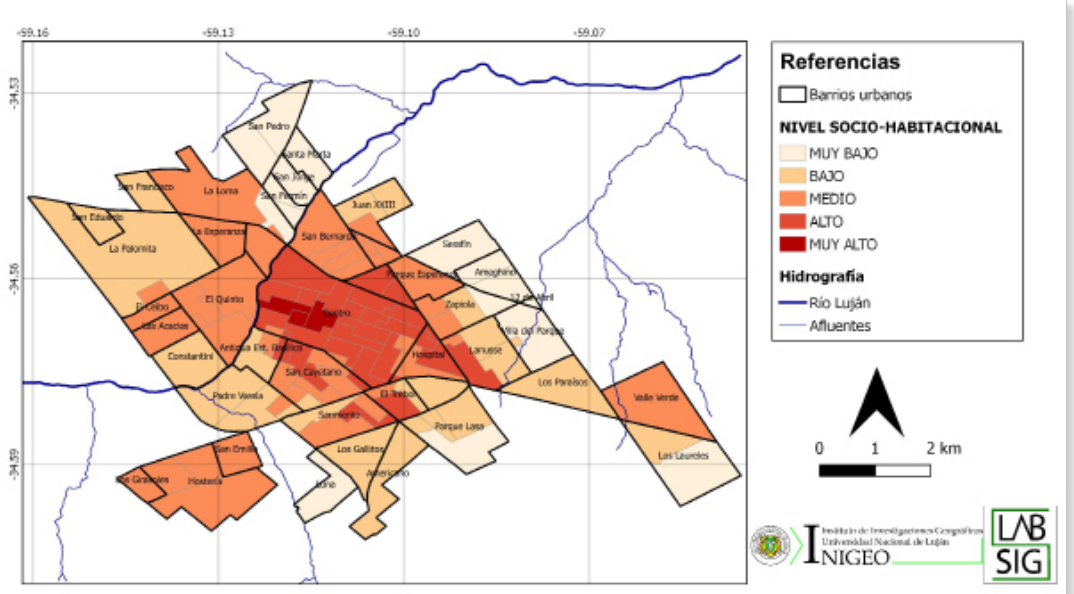

Fuente: elaboración personal con base en del Instituto Nacional de Estadística y Censos (INDEC, 2013)

Los puntajes del mapa social fueron representados cartográficamente en colores graduados considerando sus intensidades en relación directa al valor cuantitativo de las condiciones socioespaciales, las mejores en el centro se presentan en color más oscuro y las peores en la periferia aparecen en color más claro. Claramente muestra correspondencia con el modelo de ciudad latinoamericana propuesto por Griffin y Ford (1980), la de mayor aptitud para el caso de las aglomeraciones de tamaño intermedio.

El método de Linkage Analysis brindó como resultado tres macrovariables compuestas por los siguientes pares recíprocos y de las siguientes variables: (1) Macrovariable 1: (Hog_NBI-Hab_ranc: 0,88), Desc_poz, Prim_com, Sin_bl y Agua_man; (2) Macrovariable 2: (Agua_mot-Desc_cam: 0,72), Hab_cas, Rtv_prop y Rtv_oc; y (3) Macrovariable 3: (Rtv_inq-Hab_dep: 0,87), Desc_red, Agua_red, Sec_com y Ter_com. El tipo de variables asociadas permiten considerar que la Macrovariable 1 y 2 presentan una situación desfavorable y la macrovariable 3 presenta la situación favorable.

En base a los resultados obtenidos, las macrovariables 1 y 3 presentan el mayor poder de discriminación combinando variables de costo y beneficio asociadas a los puntajes más altos de correlación. El grupo 1 incluye variables de costo con un núcleo formado por las Necesidades Básicas Insatisfechas y los habitantes en viviendas tipo rancho, y el grupo 2 está compuesto por variables de beneficio con un núcleo formado por los habitantes en departamentos y el régimen de tenencia de la vivienda inquilinos. La especificidad de estos dos grupos formulada en el sentido de las variables de beneficio son consideradas para la realización del mapa social presentado en Figura 3.

Desde un punto de vista poblacional, 1.354 habitantes $(6,7 \%)$ se encuentran comprendidos en la categoría Muy Alto, 18.709 habitantes (18,9\%) en la categoría Alto, 25.085 habitantes (32\%) en la categoría Media, 20.274 (25,9\%) en la categoría Baja y 12.924 habitantes $(16,5 \%)$ en la categoría Muy Baja.

\section{ASOCIACIÓN ESPACIAL Y ÁREAS PRIORITARIAS DE PLANIFICACIÓN}

El gráfico de dispersión estandarizado muestra la asociación espacial entre la distribución espacial de la vulnerabilidad y el mapa social de la ciudad de Luján presentando una correlación de $r=-0.66$ (Figura 4). El valor negativo indica una relación inversa entre 
ambos, coincidente con los que puede apreciarse en los mapas, y el número indica una intensidad significativa de esta relación.

A medida que los valores del mapa social son más bajos, los valores de vulnerabilidad socioeconómica aumentan, siendo que el $r^{2}=0,43$ indica una relación de causalidad en la que el mapa social explica en un $43 \%$ la vulnerabilidad socioeconómica.

El modelado cartográfico realizado a partir de la combinación espacial de categorías (Figura 5) nos muestra que se encuentran representadas la totalidad de combinaciones vinculadas de manera inversa, es decir, considerando la asociación espacial entre la categoría de vulnerabilidad socioeconómica más alta y el mapa social en sus valores más bajos, y viceversa. En todos los pares de categorías inversas asociados espacialmente (Muy alto-Muy bajo, Alto-Bajo, Media-Medio, Bajo-Alto y Muy bajo-Muy alto) se detectaron unidades espaciales.

Figura 4. Diagrama de dispersión con valores estandarizados

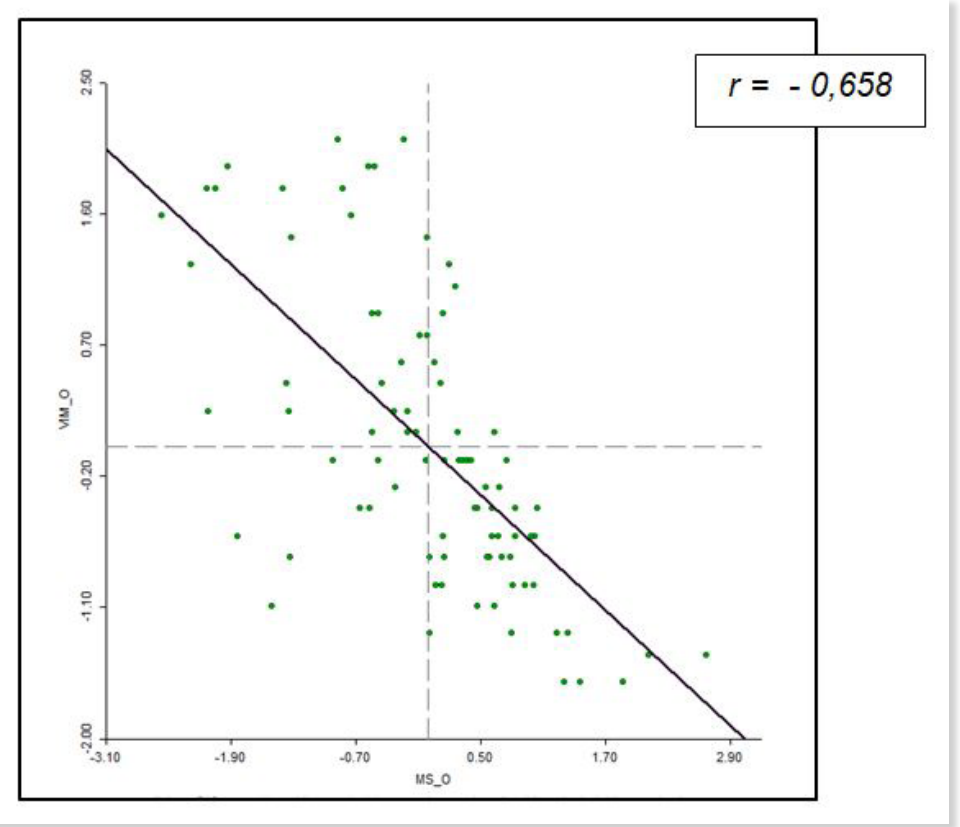

Fuente: elaboración personal

Figura 5. Áreas prioritarias de planificación en la ciudad de Luján (Buenos Aires, Argentina)

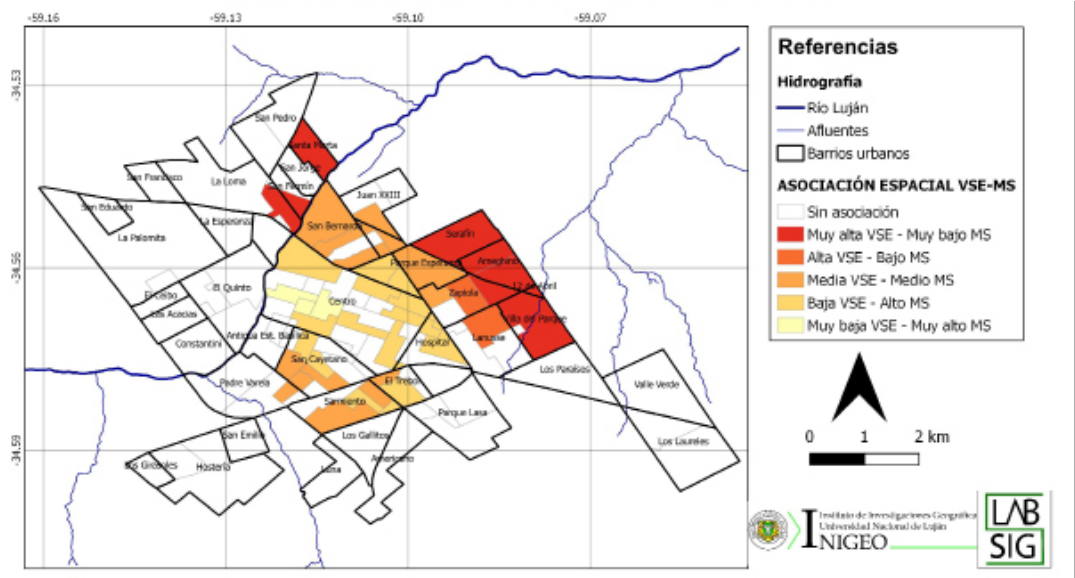

Fuente: elaboración personal con base en del Instituto Nacional de Estadística y Censos (INDEC, 2010; 2013) 
Las combinaciones de radios censales urbanos que contienen la asociación espacial entre la vulnerabilidad socioeconómica y el mapa social se presentan como áreas urbanas con diferente prioridad de planificación. De ellas presentaremos las que son consideradas áreas críticas:

Prioridad 1 (Muy alta VSE y Muy bajo MS): principalmente se encuentra en la periferia en los barrios San Fermín, Santa Marta, Serafín, Ameghino y Villa del Parque (Figura 6), concentrando 8.234 habitantes de los cuales 4.136 (50,23\%) son varones y 4.098 $(49,77 \%)$ son mujeres, en cuanto a los grupos de edad $2.930(35,58 \%)$ tienen entre 0 y 14 años de edad, 4.965 (60,30\% entre 15 y 64 años y 339 (4,12\%) de 65 y más años.

Figura 6. Ciudad de Luján. Paisaje de prioridad 1 - Muy alta VSE y Muy bajo en MS

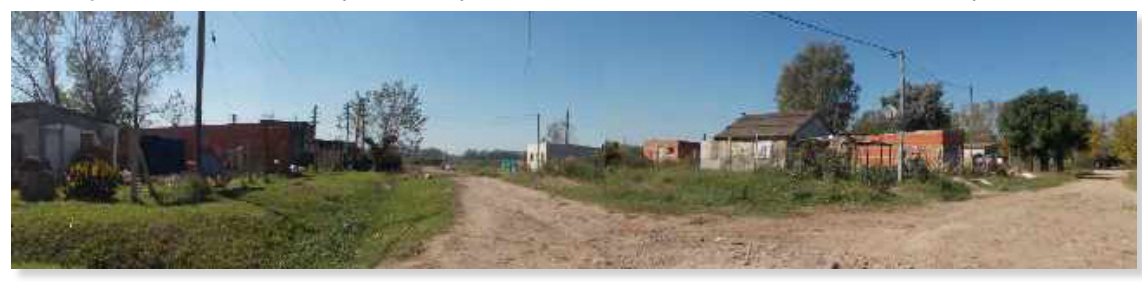

Fuente: fotografía de los autores

Prioridad 2 (Alta VSE y Bajo MS): se encuentra en una pequeña área periférica en el noreste de la ciudad correspondiente a los barrios Lanuse y Zapiola (Figura 7), concentrando 2.685 habitantes de los cuales 1.301 (48,45\%) son varones y $1.384(51,55 \%)$ son mujeres, en cuanto a los grupos de edad $770(28,68 \%)$ tienen entre 0 y 14 años de edad, 1.685 (62,76\%) entre 15 y 64 años y 230 (8,56\%) de 65 y más años.

Figura 7. Ciudad de Luján. Paisaje urbano de prioridad 2 - Alta VSE y Bajo en MS

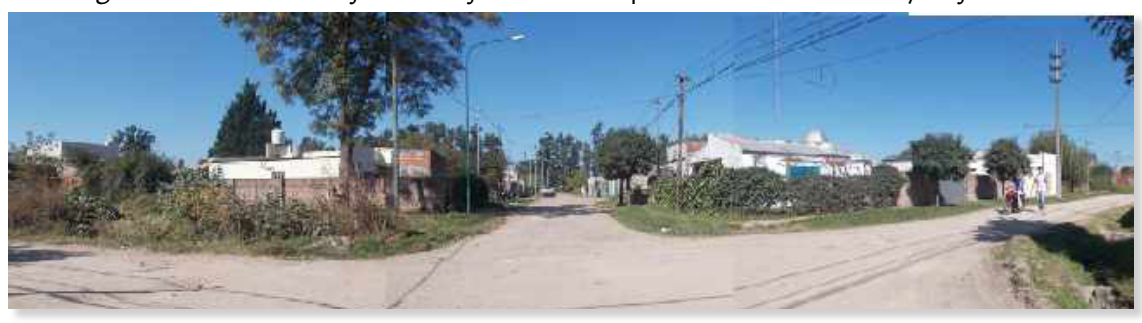

Fuente: fotografía de los autores

Valores totales (Área crítica $=$ Prioridad $1+2)$ : ubicada en la periferia extrema norte y noreste, incluye los barrios mencionados oportunamente, concentrando 10.919 habitantes de los cuales $5.437(49,79 \%)$ son varones y $5.482(50,21 \%)$ son mujeres, en cuanto a los grupos de edad 3.700 (33,89\%) tienen entre 0 y 14 años de edad, $6.650(60,90 \%)$ entre 15 y 64 años y 569 (5,21\%) de 65 y más años.

La ciudad de Luján tiene una población de 78.346 habitantes, por lo tanto, el 13,94\% están ubicados en áreas críticas y de una superficie total de 3478 hectáreas para el área urbana total, el área de prioridad 1 ocupa 301 hectáreas y el área de prioridad 2 ocupa 58 hectáreas (359 hectáreas en total, equivalente a un 10,32\% de la superficie total).

\section{CONCLUSIONES}

La confluencia entre la teoría sistémica y el análisis espacial cuantitativo con SIG permite avanzar con focalización en el estudio de la lógica social del espacio (Hillier y Han- 
son, 1984). Los conceptos de localización, distribución espacial y asociación espacial que se hacen operativos en la presente aplicación, permiten verificar de forma empírica, a partir de datos censales, la diferenciación socioespacial de la población urbana.

El mapa de la distribución espacial de las condiciones de vulnerabilidad socioeconómica de la población permitió evidenciar diferencias espaciales intraurbanas que son necesarias conocer, como parte de una etapa diagnóstica de estudio, para poder identificar aquellas áreas de la ciudad que presentarían las mayores desigualdades al momento de enfrentar situaciones peligrosas o probabilidad de ocurrencia de algún peligro, como las inundaciones, en una ciudad que recurrentemente sufre este tipo de evento.

La aplicación de metodologías del análisis multivariado brinda la posibilidad de medir la relación causal entre la distribución espacial de la vulnerabilidad socioeconómica y el mapa social de la ciudad de Luján como aproximación modelística de interpretación. Las distribuciones espaciales de ambos componentes presentan configuraciones espaciales estructurales, de correlación inversa con un porcentual de explicación. El conjunto de resultados permite identificar áreas urbanas que presentan características diferenciales como base de intervención en el ámbito de la planificación, obteniéndose aproximadamente un $10 \%$ de la superficie y un $14 \%$ de la población, con características poblacionales específicas, en la situación más desfavorecida.

La teoría sistémica y la construcción cuantitativa en procedimientos de análisis espacial con Sistemas de Información Geográfica en el marco de una Geografía Aplicada permitieron llegar a conformar distribuciones espaciales con miras a sentar bases de intervención en el marco de las prácticas del Ordenamiento Territorial, ya que los resultados obtenidos brindan apoyo técnico-científico para los organismos de planificación y gestión en relación a la toma de decisiones espaciales tendientes a mejorar las condiciones de vida de la población. Las bases de datos geográficas, la cartografía y los gráficos explicativos se presentan como herramientas factibles de ser consideradas en la toma de decisiones, en apoyo a la superación de un importante reto para el desarrollo, en este caso, descubriendo áreas prioritarias de intervención para la minimización de la vulnerabilidad poblacional.

\section{REFERENCIAS}

Barrows, H. (1923). Geography as Human Ecology. Annals of the Association of American Geographers, XIII(1), 1-14.

Bertalanffy, L. (1976). Teoría General de Sistemas (Juan Almela, trad.) México: Fondo de Cultura Económica (Obra original publicada en 1968).

Blaikie, P.; Cannon, T.; Davies, I. y Wisner, B. (2002). At risk: Natural hazards, people's vulnerability and disasters $\left(2^{\circ}\right.$ edición). London: Routedge.

Borsdorf, A. (2003). Como modelar el desarrollo y dinámica de la ciudad latinoamericana. EURE, 29(86), 37-49

Burgess, E.W. (1925). The growth of the city: an introduction to a reseach project (pp. 47-62). En R.E. Park, E.W. Burgess y R.D. McKenzie (Eds.) The City. Chicago, United States: The Chicago University Press.

Buzai, G.D. (2014). Mapas Sociales Urbanos. Buenos Aires, Argentina: Lugar Editorial.

Buzai, G.D. (2016). Urban models in the study of Latin American cities. Innsbrucker Geographische Studien, 40, 91-108. 
Buzai, G.D. (2020). Megaciudades de América Latina. Conceptos, modelos y Geografía de los procesos de estructuración urbana. Anuario de la División Geografía, 14, 1-27.

Buzai, G.D. y Baxendale, C. A. (2013). Aportes del análisis geográfico con Sistemas de Información Geográfica como herramienta teórica, metodológica y tecnológica para la práctica del ordenamiento territorial. Persona y Sociedad, 27(2), 113-141.

Buzai, G.D. y Baxendale, C.A. (2012). Análisis socioespacial con Sistemas de Información Geográfica. Tomo 2: Ordenamiento territorial / Temáticas de base vectorial. Buenos Aires, Argentina: Lugar Editorial.

Buzai, G.D. y Montes Galbán, E. (2020). Megaciudad Buenos Aires: Cartografía de su última expansión y conurbación mediante el procesamiento digital de imágenes satelitales nocturnas. Revista Cartográfica, 100, 215-238.

Buzai, G.D.; Lanzelotti, S.L.; Paso Viola, L.F. y Principi, N. (2018). Cartografía analógica y digital para la delimitación regional y el análisis temático: aplicación a la cuenca del río Luján (Argentina). Revista de Geografía Norte Grande, 69, 99-119.

Cardona, O. (1993). Evaluación de la Amenaza, la Vulnerabilidad y el Riesgo (pp. 45 65). En A. Maskrey (Ed.) Los desastres no son naturales. Bogotá, Colombia: La Red. Tercer Mundo Editores. Recuperado de http://www.planesmojana.com/documentos/estudios/19_Evaluacion\%20de\%20la\%20amenaza(1)\%200mar\%20D.\%20Cardona.pdf

Cardona, O. (2003). The need for rethinking the concepts of vulnerability and risk from Holistic Perspective: A necessary review and criticism for effective rick management (pp. 37-51). En G.F.G. Bankoff y D. Hilhosrt (Eds.) Mapping Vulnerability: Disasters, Development and People. London, England: Sterling \& VA: Earthscan

Carter, H. (1995). The Study of Urban Geography. London, England: Taylor \& Francis.

Centro Latinoamericano y Caribeño de Demografía CELADE (2002). Vulnerabilidad social y sociodemográfica: aproximaciones conceptuales, teórica, y empíricas (pp. 1-69). En CELADE (Ed.) Vulnerabilidad sociodemográfica: viejos y nuevos riesgos para comunidades, hogares y personas. Brasilia, Brasil: Publicaciones de las Naciones Unidas.

Egea Jiménez, C.; Sánchez González, D. y Soledad Suescún, J.I. (2012). Vulnerabilidad Social. Posicionamientos y ángulos desde geografías diferentes. Granada, España: Editorial Universidad de Granada.

García Castro, N. y Villerías Salinas, S. (2018). Factores socioeconómicos de vulnerabilidad de la franja costera del Estado de Guerrero, México (pp. 75-89). En S. Villerías Salinas y N. García Castro Análisis de la vulnerabilidad social desde un enfoque multidisciplinario. Ciudad de México, México: Porrúa. México.

García de León, A. (1989). La metodología del Valor Índice Medio. Boletín del Instituto de Geografía UNAM, 9, 69-87.

García de León, A. (1997). Empleo de una metodología multivariada para la clasificación de unidades territoriales. Geografía y Desarrollo, 14, 5-20.

García, R. (2006). Sistemas Complejos. Barcelona, España: Gedisa.

Gottdiener, M.; Budd, L. y Lehlovuori, P. (2016). Key concepts in Urban Studies. London, England: SAGE.

Griffin, E. y Ford, L. (1980). A model of Latin american city structure. The Geographical Review, 70(4), 397-422.

Harris, C.D. y Ullman, E.L. (1945). The Nature of Cities. The Annals of the American Aca- 
demy of Political and Social Sciences, CCXLII, 7-17.

Herzer, H. (1990). Los desastres no son tan naturales como parecen. Medio Ambiente y Urbanización, año 8, (30).

Hillier, B. y Hanson, J. (1984). The Social Logic of Space. Cambridge, England: Cambridge University Press.

Hoyt, H. (1939). The Structure and Growth of Residential Neighborhoods in American Cities. Washington D.C., United States: Washington Federal Housing Administration.

Humacata, L. (2015). Análisis espacial con Sistemas de Información Geográfica aplicado al eje de crecimiento oeste de la Región Metropolitana de Buenos Aires (pp. 179-208). En S. Vidal Koppmann (Comp.) Metrópolis en Mutación. Buenos Aires, Argentina: Café de las Ciudades.

Instituto Nacional de Estadística y Censos, INDEC. (2013). Censo Nacional de Población, Hogares y Vivienda 2010. Base de datos REDATAM. Instituto Nacional de Estadística y Censos de la República Argentina. Buenos Aires, Argentina.

Janoschka, M. (2002). El nuevo modelo de la ciudad latinoamericana: fragmentación y privatización. EURE, 28(85), 11-29.

Kates, R.W. (1971). Natural Hazard in Human Ecological Perspective: Hypotheses and Models. Economic Geography, 47(3), 438-451.

Lavell, A. (1996). Degradación ambiental, riesgo y desastre urbano: problemas y conceptos; hacia la definición de una agenda de investigación (pp. 2-30). En M.A. Fernández (Comp.) Ciudades en riesgos: degradación ambiental, riesgos urbanos y desastres. Lima: La Red. Recuperado de https://www.desenredando.org/public/libros/1996/cer/ CER_cap02-DARDU_ene-7-2003.pdf

Martínez Rubiano, M.T. (2009). Los geógrafos y la teoría de riesgos y desastres ambientales. Perspectiva Geográfica, 14, 241-263.

McQuitty, L.L. (1957). Elementary Linkage Analysis for the Isolation of Orthogonal and Oblique Types and Typal Relevancies. Educational and Psychological Measurement, 17, 207-229 Phlipponneau, M. (2001). Geografía Aplicada. Barcelona, España: Ariel.

Principi, N. (2018). Propuesta teórico-metodológica para el análisis de riesgo y vulnerabilidad social ante la amenaza de inundaciones en la ciudad de Luján (Buenos Aires, Argentina) (pp. 75-89). En S. Villerías Salinas y N. García Castro Análisis de la vulnerabilidad social desde un enfoque multidisciplinario. Ciudad de México, México: Porrúa. México.

Principi, N. (2020). El enfoque sistémico en el análisis de riesgos en Geografía. Anuario de la División Geografía, 14, 1-8.

Racine, J.B. y Reimond, H. (1973). L'analyse quantitative en géographie. Paris, France: Presses Universitaires de France.

White, G. (1974). Natural Hazards: Local National, global. Oxford, England: Oxford University Press.

Wilches Chaux, G. (1993). La Vulnerabilidad Global (pp. 11-44). En A. Maskrey (Ed.) Los desastres no son naturales. Bogotá, Colombia: La Red.Tercer Mundo Editores.

Noelia Principi es Profesora en Geografía, Licenciada en Geografía, Especialista en Teledetección y Sistemas de Información Geográfica aplicados al estudio del medio ambiente, Magister profesional en Sistemas de Información Geográfica Teledetección y Doctoranda en Geografía. Estadías de formación académica en el Instituto de Hidrología de 
Llanuras UNCPB (Argentina) y en la Escuela de Geografía UNA (Costa Rica). En la Universidad Nacional de Luján es Profesora del Departamento de Ciencias Sociales, Becaria de Formación Superior y Coordinadora del Laboratorio de Análisis Espacial y Sistemas de Información Geográfica (LabSIG). Instituto de Investigaciones Geográficas. Universidad Nacional de Luján. Ruta Nacional N 5 y Av. Constitución, (6700) Luján, Buenos Aires, Argentina, nprincipi@unlu.edu.ar, ORCID https://orcid.org/0000-0002-8819-6743

Gustavo Buzai es Profesor de Geografía, Licenciado en Geografía y Doctor en Geografía. Es Investigador Principal del Consejo Nacional de Investigaciones Científicas y Técnicas (CONICET) Estadías de formación académica en el Centro de Ecología UFRGS (Brasil) y de investigación posdoctoral en el Departamento de Geografía UAM (España) y en el Institut für Geographie UIBK (Austria). En la Universidad Nacional de Luján es Profesor del Departamento de Ciencias Sociales, Investigador del Consejo Nacional de Investigaciones Científicas y Técnicas (CONICET), Director del Programa de Docencia e Investigación en Sistemas de Información Geográfica (PRODISIG) y Director del Instituto de Investigaciones Geográficas (INIGEO). Instituto de Investigaciones Geográficas. Universidad Nacional de Luján. Ruta Nacional N 5 y Av. Constitución, (6700) Luján, Buenos Aires, Argentina, gdb@unlu.edu.ar, ORCID https://orcid.org/0000-0003-4195-5324 